\title{
A Study on Unified Testing Method for IEC 61850 Based Server with Focus on Setting Group Function
}

\author{
Namdae Kim, Alidu Abubakari, Byungtae Jang \\ Power System Technology Group, Korea Electric Power Corporation (KEPCO), Daejeon, South Korea \\ Email: namdae.kim@kepco.co.kr, aabubakari32@yahoo.com, byungtae.jang@kepco.co.kr
}

How to cite this paper: Kim, N., Abubakari, A. and Jang, B. (2018) A Study on Unified Testing Method for IEC 61850 Based Server with Focus on Setting Group Function. Smart Grid and Renewable Energy, 9, 186-198.

https://doi.org/10.4236/sgre.2018.910012

Received: September 7, 2018

Accepted: October 23, 2018

Published: October 26, 2018

Copyright $\odot 2018$ by authors and Scientific Research Publishing Inc. This work is licensed under the Creative Commons Attribution International License (CC BY 4.0).

http://creativecommons.org/licenses/by/4.0/

\begin{abstract}
The digital substation system consists of an Intelligent Electronic Device (IED) which requires information for the supervision, protection, and control, as well as a client operating system for substation operation. IEC 61850 communication standard has been defined for information exchange between heterogeneous digital devices in such substations. Currently, the Korea Electric Power Corporation (KEPCO) digital substation system uses unbuffered reporting and Generic Object Oriented Substation Events (GOOSE) communication functions for monitoring, protection and control automation. However, it is expected that the Setting Group function will be needed to operate adaptive intelligent substation system. The Setting Group function is advantageous in that it is possible to change the setting of the IED in one unified way through the client operating system, rather than changing the correction value of an IED through different manufacturer-based engineering tools. In order to utilize the Setting Group function of the IED in real substation scenario, it is necessary to test both the communication function with the host operating device and the electric functional test to ensure that correction value is properly changed. In this paper, we introduce the IEC 61850 Setting Group function, and propose a method that can integrate communication function and electrical function test of an IED conforming to the setting group functionality. We also tested the Setting Group function of the actual IED device through the proposed integrated functional test method and analyzed the results. We were able to simplify the process of testing both the electrical and communication signals in digital substations.
\end{abstract}

\section{Keywords}

IEC 61850, Substation Automation, IED, Function Test, Setting Group, Conformance Testing, Electrical Testing, Unified Testing, UCAIUG 


\section{General Introduction}

In recent times, KEPCO has been researching and constructing digital substations based the globally accepted IEC 61850 communication standard. Also traditional analog substations are undergoing the digitalization phase by replacing and retrofitting old electro-mechanical relays with newer IED devices together running over modern Ethernet technologies and communicating via the same IEC 61850 standard. The typical digital substation system consists of an IED that obtains data from primary equipment and also provides information for the purposes of supervision, protection, and control, as well as a client device which contains the parent operating system for actual substation operation [1] [2]. IEC 61850 communication standard is an internationally accepted standard for the exchange of information between heterogeneous digital devices (IEDs) in digital substations [3] [4] [5]. The revised standard (edition 2) has extended this functionality by expanding the application domain from the substation to micro grid, wind power etc. [6] [7]. Currently, KEPCO's digital substation system uses Unbuffered Reporting and Generic Object Oriented Substation Events (GOOSE) communication functions for monitoring, protection and control automation. However, with the implementation of Thyristor Controlled Series Compensation (TCSC) and High Voltage Direct Current (HVDC), it is expected that the IEC 61850 Setting Group function will be needed to operate the adaptive intelligent substation system. The advantage of using the Setting Group function is that it is possible to change the setting of the IED in one unified way through the client operating device, rather than changing it through the use of different manufacturer specific engineering tool [8]. The first step in adopting any IEC 61850 communication service is to perform the communication/conformance test [9] [10] [11]. The communication function test of the IED can be implemented through the server conformance test procedure defined by the Utility Communication Architecture International Users Group (UCAIUG) specification, but there is a limit to check whether the actual setting is changed or not [12]. Thus in order to test the Setting Group function, it is necessary to perform an electrical functional test together with the communication test using host operating device to ascertain whether the setting actually changed within the IED is changed. In this paper, we introduce the setting group function presented in IEC 61850, and propose an algorithm that can integrate the communication test and electrical function test in an IED. We also implemented the proposed integrated functional test method on a practical IED and the results were analyzed. This paper is organized as follows; Section 2 introduces the IEC 61850 Setting Group function. Section 3 presents the proposed Setting Group functionality test method. Section 4 then proceeds to implement the proposed test case using the KEPCO server conformance test tool. Section 5 discusses the results for both the basic communication and the electrical test case. We finally present our concluding thoughts in Section 6 .

\section{Introduction to the IEC 61850 Setting Group Function}

The method of IED configuration setting used in the digital substation system 
requires the use of manufacturer specific engineering tool for each IED under consideration. Example includes using DIGSI for engineering only Siemens IED devices while using PCM600 for only ABB devices. This means the system integration engineer needs to understand all these manufacturer specific engineering tools. In a heterogeneous substation with multiple multi-vendor devices, this is not only very hectic to implement changes but also might be prone to errors which can be hazardous in a substation setting. On the other hand, by using the IEC 61850 Setting Group function [8], it is possible to change the setting of the IED in a unified way through the parent operating system of a single client device. Especially, it can be applied to the adaptive intelligent substation system operation where there is the need to change values quickly due to fact that the using the Setting Group functionality does not require complete re-engineering of the IED and can be supported by any vendor. Figure 1 shows the Setting Group functionality as it's applied to PVOC (overvoltage protection) and PDIF (differential protection) logical nodes (LN) [13]. The Setting Group functionality spans across multiple logical nodes, therefore various data attributes are selected as the settings data across the PVOC and PDIF LNs. Ideally the Setting Group Control Block (SGCB) which is used to control the read/write actions on the Setting Group is stored in Logical node zero (LLNO). Data can be written or read from the settings data in the active buffer and each setting group contains a consistent set of values [8] [13].

\subsection{Application of the Setting Group Function in IEDs}

The Setting Group Control Block (SGCB) is a logical node which manages the setting data of the IED as explained above. As can be observed in Table 1, it consists of five attributes (5). The NumOfSG is a fixed value which represents the total number of Setting groups in a single IED device. Within the range of NumOfSG value, the stored value can be changed using the EditSG attribute.

The configuration of the Setting Group functionality in the practical IED used for this paper is represented in Figure 2. The IEC 61850 standard provides the following six services for the Setting Group function as presented in Table 2, and the situation in which each service is applied to the actual IED is shown in Figure 3(a). For example, you can edit the settings by first using the SelectEditSG service and then change the setting value of an actual IED to the selected Setting Group using the SelectActiveSG service.

Table 1. Setting group basic attributes.

\begin{tabular}{cc}
\hline Property & Meaning \\
\hline NumOfSG & Setting Group number \\
ActSG & Currently set Setting Group \\
EditSG & Setting Group you want to edit \\
CnfEdit & Commit Setting Group value to edit command \\
LActTm & ActSG to Setting Group last selected time \\
\hline
\end{tabular}


Table 2. Setting group basic service attributes.

\begin{tabular}{cc}
\hline $\begin{array}{c}\text { Communication } \\
\text { Service }\end{array}$ & Meaning \\
\hline GetSGCBValues & Service to read SGCB settings \\
Service to read data attribute value of \\
FC $=$ SG of Logical Node belonging \\
to the setting group of ActSG
\end{tabular}

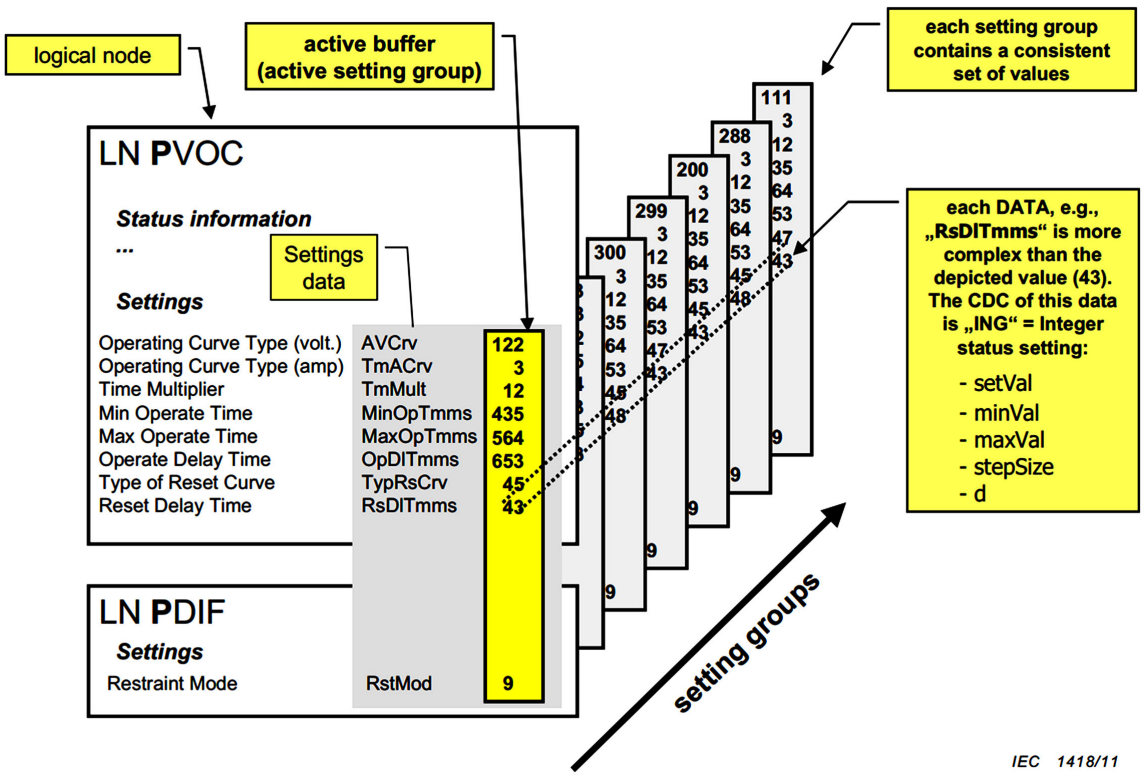

Figure 1. Setting group concept [13].

\section{D101_C6100PROT/LLNO.SGCB}

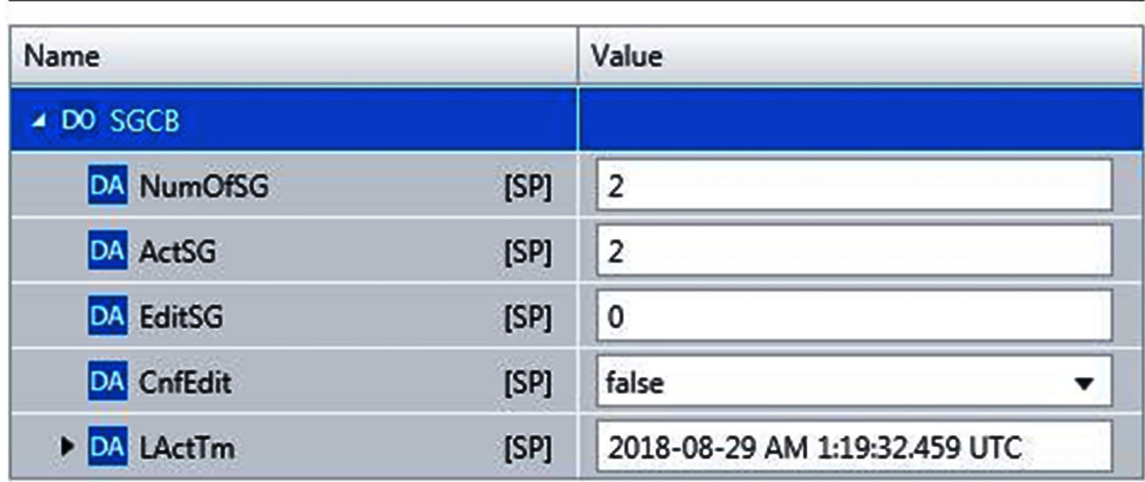

Figure 2. Setting group concept. 


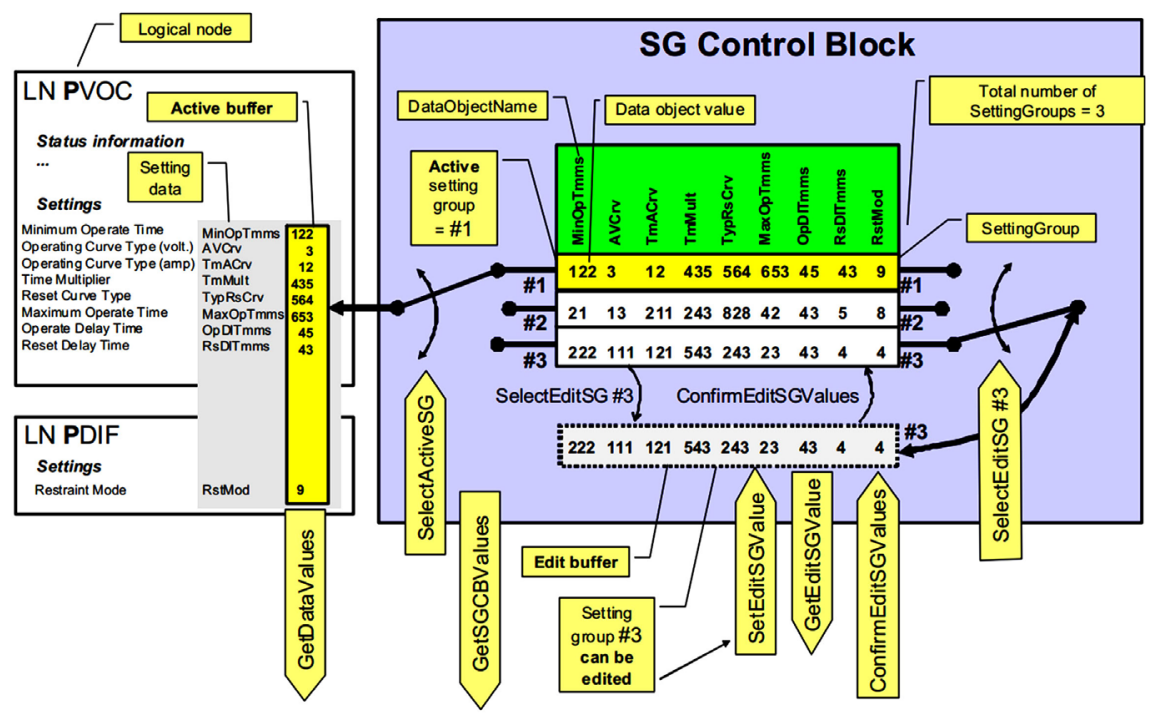

(a)

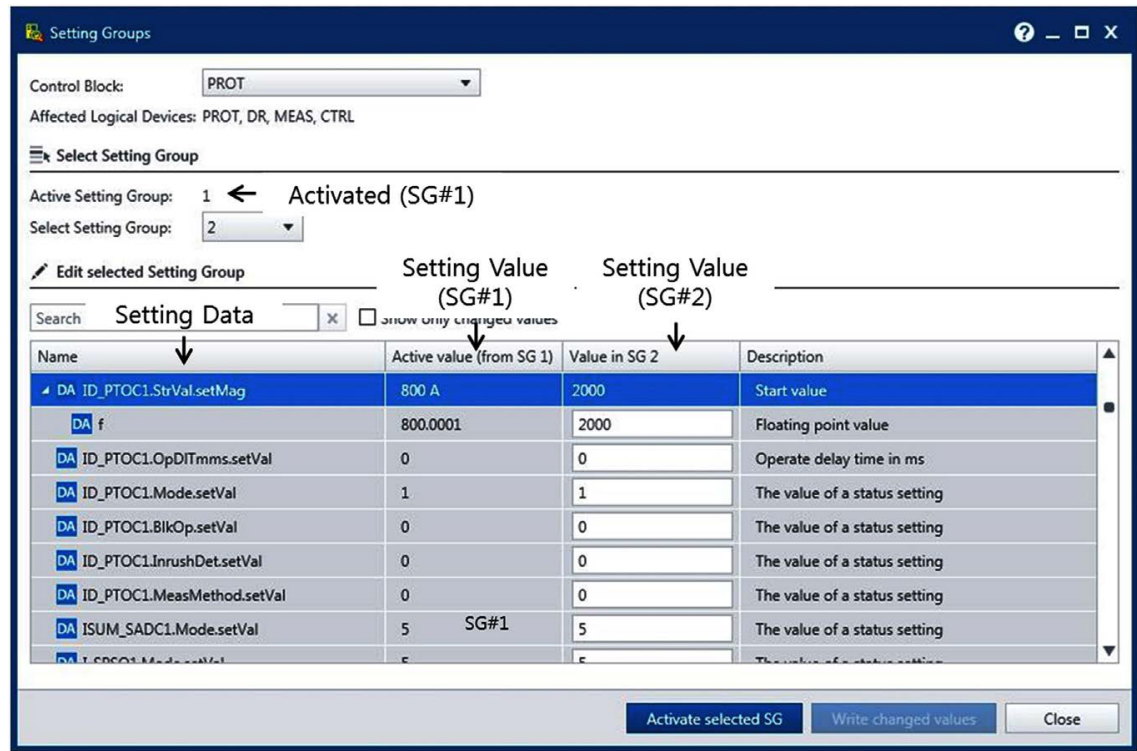

(b)

Figure 3. (a) Summary of IEC 61850 Setting Group Service functionality [14], (b) Application of Setting Group function in IED Information model.

In Figure 3(b), the actual IED Setting group 1 ( $(\mathrm{GG} \# 1)$ data is represented using the Omicron IEDScout software.

\subsection{Setting Group Function Server Conformance Test}

In order to test IEC 61850-based Setting Group functions, it is necessary to confirm that they respond appropriately to service requests from higher-level client operating systems. All devices which communicate using the IEC 61850 standard are expected to conform to the test procedures standardized by the Utility Communication Architecture International Users Group (UCAIUG). An example of such as test procedure is the sSg3 shown in Figure 4. The main reason for 
standardizing the test procedure is to enable globally acceptable and reproducible results. The client uses the parent operating system to request a communication service from the IED according to the predetermined procedure and the IED responds to the requested service. When the IED performs a service response according to a predetermined procedure, it is subjected to conformity authentication for the service. Therefore, as can be seen in Figure 4, the client requests a series of Setting Group services including SelectActiveSG, GetSGCBValues and GetDataValues for different NumOfSG. It is expected that the Device Under Test (DUT) would respond positively to each service request. In case the DUT responds with a service error, it is assumed that the device does not conform well to the IEC 61850 standard. Further analysis of the communication packet is required to observe the communication error in detail.

\section{Proposed Setting Group Function Test Method}

\subsection{Advantages of the Proposed Integrated Functional Test}

To test the Setting Group function of an intelligent electronic device, it is necessary to test not only the communication service test but also whether or not the electrical signal is actually applied to the IED. The communication service test can be tested through the server conformance test procedure defined in the UCAIUG specification, but there is a limit in checking whether the actual setting is changed or not. Simulation devices capable of performing integrated functional testing of the IED devices have been devised, but actual functional testing methods have not been formalized. That is the procedure for implementing a functional test involving both the electrical and communication conformance test has not yet been standardized. Through the integrated test method proposed in this paper, it is possible to judge the suitability of the Setting Group function by deriving the reproducible results in testing the communication function and the electric function of an IEC 61850 Based server device (IED).

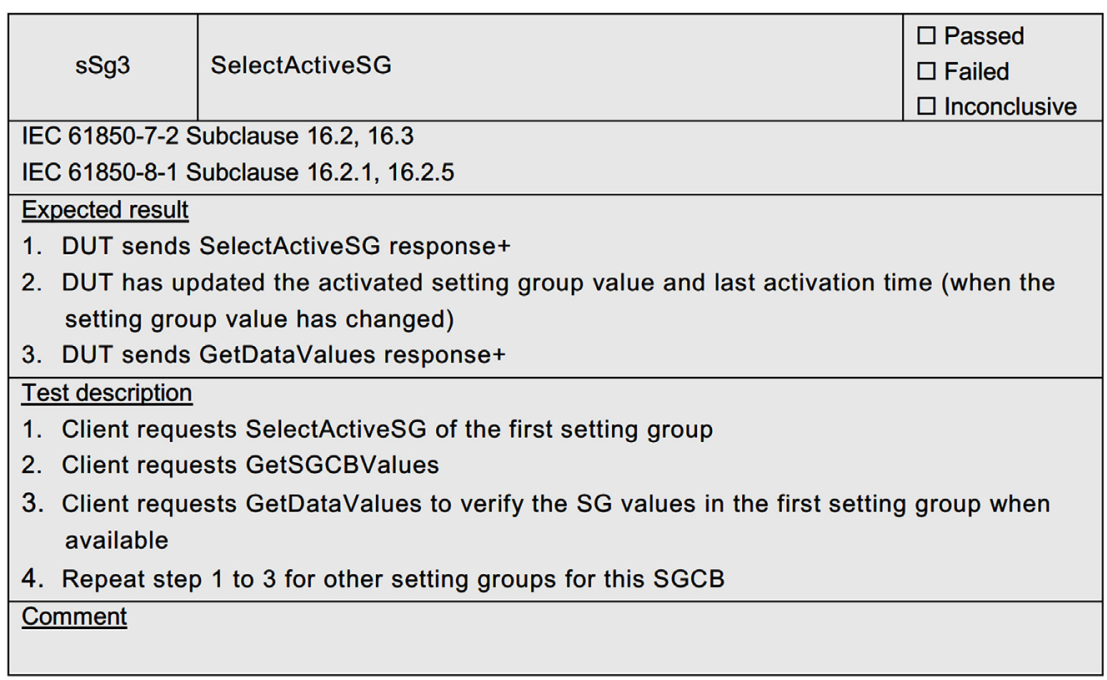

Figure 4. Standard communication service for IEC 61850 Setting Group service [15]. 


\subsection{Proposed Integration Function Test Procedure}

The procedure of the proposed Setting Group integrated testing function can be summarized as shown in Table 3. This was achieved by modifying the standard sSg3 proposed to test the Setting Group communication function by UCAIUG. The UCAIUG sSg3 test is used to verify that the IED device under test responds normally to various requested services when the client operating device requests to read or write values to/from the Setting Group. The integrated functional test procedure therefore adds an additional procedure which applies an electrical signal to the Setting Group after the changes have been made by the communication service. This would enable the test engineer to verify whether the applied Setting Group changes can affect actual or practical substation performance. At this time, the active Setting Group should include setting data that can reacted to the electric signal to produce both positive and negative action depending on the circumstance. A functional test flow chart combining the communication test and the electrical test can be shown in Table 3. Figure 5 shows the flow diagram of the proposed unified testing procedure.

Table 3. Proposed procedure for Setting Group functional test.

\section{Test procedure}

1. The Client requests the SelectActiveSG to set the DUT as the first Setting Group.

2. Client requests GetSGCBValues from the DUT.

The client requests the GetDataValues of the SG data to check the value of the first Setting Group set in Step 1 to the DUT.

4. The Tester applies electrical signal to the DUT to confirm the application of Setting Group $\# 1$.

5. The client requests the SelectActiveSG to set the DUT as the second Setting Group.

6. Client requests GetSGCBValues from the DUT.

The client requests the GetDataValues of the SG data to check the value of the second Setting Group set in Step 1 to the DUT.

The Tester applies the same electrical signal to the DUT to confirm the application of Setting Group \#2.

\section{Expected Results}

1. The DUT responds with SelectActiveSG response +.

2. The DUT responds with a GetSGCBValues response + with the same value set in Step 1.

3. The DUT responds with GetDataValues response +.

4. The DUT operates normally.

5. The DUT responds with SelectActiveSG response +.

6. The DUT responds with a GetSGCBValues response + with the same value set in Step 5.

7. The DUT responds with GetDataValues response +.

8. DUT is inactive.

Comment

Input Signal: Setting Data (SG\#1) < Input Signal < Setting Data (SG\#2) 


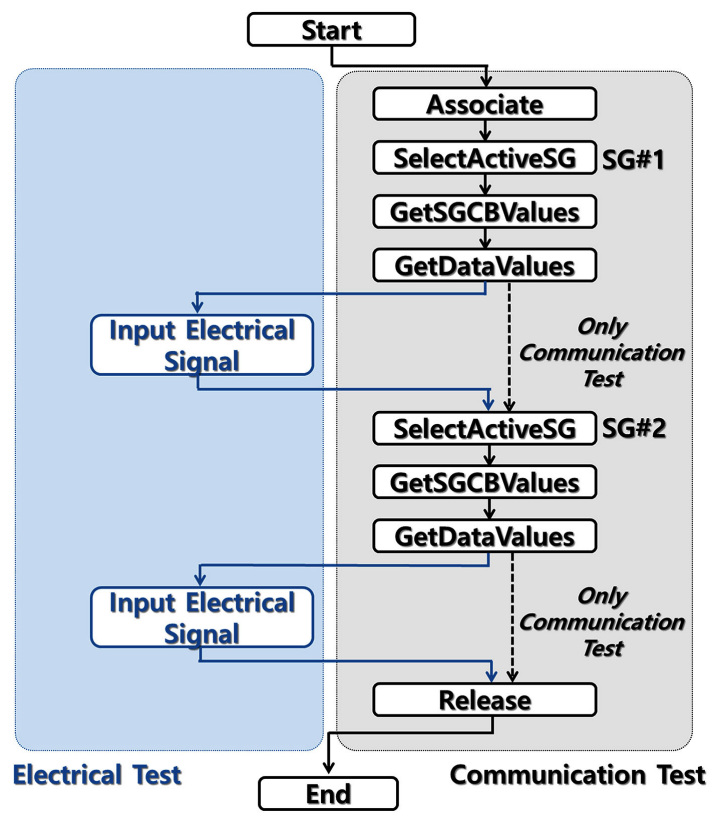

Figure 5. Flowchart for the integrated functional test.

\section{Setting Group Integration Function Test}

The configuration for testing the Setting Group function in an IED is shown in Figure 6.

The client simulator that requests the setting group service to the test object is the server suitability test tool developed by KEPCO and Omicrons CMC256 is the device simulator that is used to supply the current. The setting data is selected as the minimum operating current value in order to confirm the electric static and dynamic reaction according to the setting group change of the DUT and as can be seen in Table 4 the setting data value of each setting group is set to 800 [A] and 2000 [A]. The current application simulator applied 1600 [A], which is the value between each setting data value, so that the DUT can judge the electric static behavior as shown in Figure 7. The PTOC1.StrVal.setMag.f represents the minimum operating current for the overcurrent protection logical node.

\section{Test Results and Analysis}

\subsection{Setting Group Communication Function Test Result}

The test procedure related to the communication function test during the setting group integration function test is 1 - 3 for Setting Group No. 1 (SG \#1) and 5 - 7 for Setting Group No. 2 (SG \#2). This implies that NumOfSG $=2$. The results of requesting SelectActiveSG, GetSGCBValues, and GetDataValues services from the IED using the server conformance test tool and based on the UCAIUG procedure are shown in the Figure 8(a) and Figure 8(b) using the Omicron IEDScout packet analyzer. SG \#1 is activated using the SelectActiveSG as seen in Figure $8(a)$. The result is successful as can be observed in the response from the 
DUT.

The setting data of 800 [A] is checked as shown in Figure 8(b). Also, when SG $\# 2$ is activated in Figure 8 (c), the setting data value of 2000 [A] is confirmed as shown in Figure 8(d). Ideally this implies that the DUT has responded successfully to the requests of the client (server testing device) and has therefore successfully passed the test case (sSg3). This is not enough to ascertain the function of the Setting Group in a real substation setting, thus we propose the combined communication and electrical test procedure below which would ensure not only the communication is correctly implemented but also the output of the electrical signal input is correctly implemented and observed.

\subsection{Setting Group Electrical Function Test Result}

The test procedure related to the electrical function test during the setting group integration function test corresponds to No. 4 (SG \#1) and No. 8 (SG \#2) from Table 3. When the operation current of 1600 [A] is applied to the IED using the current application simulator in this case CMC256, it is confirmed that the protection element of the IED operates as shown in Figure 9(a). Also, the SG \#2 is activated under the condition that the same operating value of 1600 [A] is applied, it is confirmed that the protection element is non-operational as shown in Figure 9(b). Results 9a and 9b are presented using the Siemens SIGRA 4 in order to observe the overcurrent tripping even clearly when the applied current exceeds the setting group PTOC1.StrVal.setMag.f value.

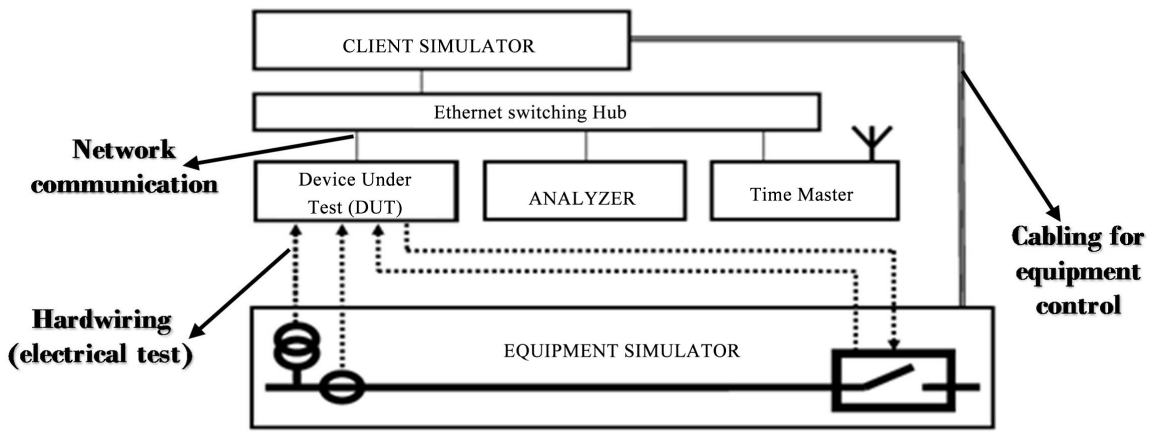

Figure 6. Setting group integration function test configuration [15].

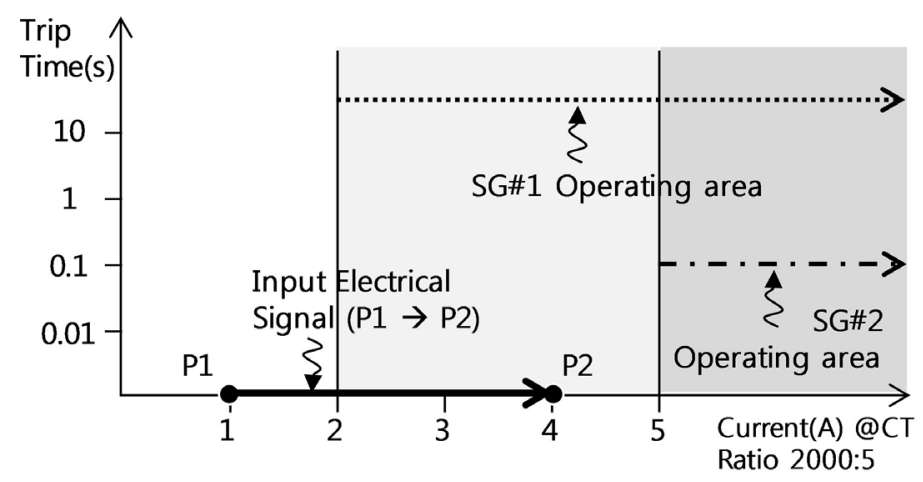

Figure 7. Characteristic curve for Overcurrent operation in IED. 


\begin{tabular}{|c|c|c|c|}
\hline Source & Destination & \multicolumn{2}{|c|}{ Description } \\
\hline 192.168.10.254 & 192.168 .10 .60 & \multicolumn{2}{|c|}{ Associate with 192.168 .10 .60} \\
\hline 192.168.10.254 & 192.168.10.60 & \multicolumn{2}{|c|}{ GetSGCBValues of D101_C6100PROT/LLNO.SGCB.NumOfSG } \\
\hline 192.168.10.254 & 192.168 .10 .60 & \multicolumn{2}{|c|}{ GetSGCBValues of D101_C6100PROT/LLN0.SGCB } \\
\hline 192.168.10.254 & 192.168 .10 .60 & \multicolumn{2}{|c|}{ SelectActiveSG of D101_C6100PROT/LLNO.SGCB.ActSG } \\
\hline 192.168.10.254 & 192.168.10.60 & \multicolumn{2}{|c|}{ GetSGCBValues of D101_C6100PROT/LLN0.SGCB } \\
\hline 192.168.10.254 & 192.168 .10 .60 & \multicolumn{2}{|c|}{ GetDataValues of D101_C6100PROT/ID_PTOC1.StrVal.setMag.f } \\
\hline 192.168.10.254 & 192.168 .10 .60 & \multicolumn{2}{|c|}{ GetSGCBValues of D101_C6100PROT/LLNO.SGCB } \\
\hline 192.168.10.254 & 192.168.10.60 & \multicolumn{2}{|c|}{ SelectActiveSG of D101_C6100PROT/LLNO.SGCB.ActSG } \\
\hline 192.168.10.254 & 192.168.10.60 & \multicolumn{2}{|c|}{ GetSGCBValues of D101_C6100PROT/LLNO.SGCB } \\
\hline 192.168.10.254 & 192.168.10.60 & \multicolumn{2}{|c|}{ GetDataValues of D101_C6100PROT/ID_PTOC1.StrVal.setMag.f } \\
\hline 192.168.10.254 & $|192.168 .10 .60|$ & \multicolumn{2}{|c|}{ Release with 192.168 .10 .60} \\
\hline \multicolumn{3}{|l|}{$\Delta$ Request } & $\triangle$ Response \\
\hline \multicolumn{3}{|c|}{ 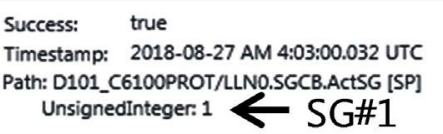 } & $\begin{array}{l}\text { Success: true } \leftarrow \text { Changed SG\#1 } \\
\text { Timestamp: } 2018-08-27 \text { AM 4:03:00.117 UTC } \\
\text { WriteResults: } \\
\text { Success }\end{array}$ \\
\hline
\end{tabular}

(a)

\begin{tabular}{|c|c|c|c|}
\hline Source & Destination & \multicolumn{2}{|l|}{ Description } \\
\hline 192.168.10.254 & 192.168.10.60 & \multicolumn{2}{|c|}{ Associate with 192.168 .10 .60} \\
\hline 192.168.10.254 & 192.168.10.60 & \multicolumn{2}{|c|}{ GetSGCBValues of D101_C6100PROT/LLNO.SGCB.NumOfSG } \\
\hline 192.168.10.254 & 192.168.10.60 & \multicolumn{2}{|c|}{ GetSGCBValues of D101_C6100PROT/LLNO.SGCB } \\
\hline 192.168.10.254 & 192.168 .10 .60 & \multicolumn{2}{|c|}{ SelectActiveSG of D101_C6100PROT/LLNO.SGCB.ActSG } \\
\hline 192.168.10.254 & 192.168.10.60 & \multicolumn{2}{|c|}{ GetSGCBValues of D101_C6100PROT/LLNO.SGCB } \\
\hline 192.168.10.254 & 192.168 .10 .60 & \multicolumn{2}{|c|}{ GetDataValues of D101_C6100PROT/ID_PTOC1.StrVal.setMag.f } \\
\hline 192.168.10.254 & 192.168 .10 .60 & \multicolumn{2}{|c|}{ GetSGCBValues of D101_C6100PROT/LLNO.SGCB } \\
\hline 192.168.10.254 & 192.168 .10 .60 & \multicolumn{2}{|c|}{ SelectActiveSG of D101_C6100PROT/LLNO.SGCB.ActSG } \\
\hline 192.168.10.254 & 192.168.10.60 & \multicolumn{2}{|c|}{ GetSGCBValues of D101_C6100PROT/LLNO.SGCB } \\
\hline 192.168.10.254 & 192.168.10.60 & \multicolumn{2}{|c|}{ GetDataValues of D101_C6100PROT/ID_PTOC1.StrVal.setMag.f } \\
\hline 192.168.10.254 & 192.168.10.60 & \multicolumn{2}{|c|}{ Release with 192.168 .10 .60} \\
\hline \multirow{2}{*}{\multicolumn{3}{|c|}{$\begin{array}{ll}\text { S Request } & \text { Setting Data Ref. } \\
\text { Success: } & \text { true } \\
\text { Timestamp: } & \text { 2018-08-27 AM 4:0我4.077 UTC }\end{array}$}} & $\triangle$ Response \\
\hline & & & $\begin{array}{l}\text { Success: } \quad \text { true } \\
\text { Timestamp: } 2018-08-27 \text { AM 4:03:14.092 UTC } \\
\text { Values: } \\
\quad \text { Float: } 800.0001 \leftarrow \text { Setting Value }\end{array}$ \\
\hline
\end{tabular}

(b)

\begin{tabular}{|c|c|c|c|}
\hline Source & Destination & \multicolumn{2}{|c|}{ Description } \\
\hline 192.168.10.254 & 192.168 .10 .60 & \multicolumn{2}{|c|}{ Associate with 192.168.10.60 } \\
\hline 192.168.10.254 & 192.168 .10 .60 & \multicolumn{2}{|c|}{ GetSGCBValues of D101_C6100PROT/LLNO.SGCB.NumOFSG } \\
\hline 192.168.10.254 & 192.168.10.60 & \multicolumn{2}{|c|}{ GetSGCBValues of D101_C6100PROT/LLNO.SGCB } \\
\hline 192.168.10.254 & 192.168 .10 .60 & \multicolumn{2}{|c|}{ SelectActiveSG of D101_C6100PROT/LLNO.SGCB.ActSG } \\
\hline 192.168.10.254 & 192.168.10.60 & \multicolumn{2}{|c|}{ GetSGCBValues of D101_C6100PROT/LLNO.SGCB } \\
\hline 192.168.10.254 & 192.168 .10 .60 & \multicolumn{2}{|c|}{ GetDataValues of D101_C6100PROT/ID_PTOC1.StrVal.setMag.f } \\
\hline 192.168.10.254 & 192.168.10.60 & \multicolumn{2}{|c|}{ GetSGCBValues of D101_C6100PROT/LLNO.SGCB } \\
\hline 192.168.10.254 & 192.168.10.60 & \multicolumn{2}{|c|}{ SelectActiveSG of D101_C6100PROT/LLNO.SGCB.ActSG } \\
\hline 192.168.10.254 & 192.168 .10 .60 & \multicolumn{2}{|c|}{ GetSGCBValues of D101_C6100PROT/LLN0.SGCB } \\
\hline 192.168.10.254 & 192.168 .10 .60 & \multicolumn{2}{|c|}{ GetDataValues of D101_C6100PROT/ID_PTOC1.StrVal.setMag.f } \\
\hline 192.168.10.254 & 192.168.10.60 & \multicolumn{2}{|c|}{ Release with 192.168 .10 .60} \\
\hline \multicolumn{3}{|l|}{$\triangle$ Request } & $\Delta$ Response \\
\hline \multicolumn{3}{|c|}{$\begin{array}{l}\text { Success: } \quad \text { true } \\
\text { Timestamp: 2018-08-27 AM 4:03:16.193 UTC } \\
\text { Path: D101_C6100PROT/LLN0.5GCB.ACtSG [SP] } \\
\text { UnsignedInteger: } 2 \text { SG\#2 }\end{array}$} & $\begin{array}{l}\text { Success: } \quad \text { true } \text { Changed SG\#2 }_{\text {Timestamp: }} \text { 2018-08-27 AM 4:03:16.281 UTC } \\
\text { WriteResults: } \\
\quad \text { Success }\end{array}$ \\
\hline
\end{tabular}

(c) 


\begin{tabular}{|c|c|c|c|}
\hline Source & Destination & \multicolumn{2}{|l|}{ Description } \\
\hline 192.168.10.254 & 192.168 .10 .60 & \multicolumn{2}{|c|}{ Associate with 192.168 .10 .60} \\
\hline 192.168.10.254 & 192.168 .10 .60 & \multicolumn{2}{|c|}{ GetSGCBValues of D101_C6100PROT/LLNO.SGCB.NumOFSG } \\
\hline 192.168.10.254 & 192.168.10.60 & \multicolumn{2}{|c|}{ GetSGCBValues of D101_C6100PROT/LLNO.SGCB } \\
\hline 192.168.10.254 & 192.168 .10 .60 & \multicolumn{2}{|c|}{ SelectActiveSG of D101_C6100PROT/LLNO.SGCB.ActSG } \\
\hline 192.168.10.254 & 192.168 .10 .60 & \multicolumn{2}{|c|}{ GetSGCBValues of D101_C6100PROT/LLNO.SGCB } \\
\hline 192.168.10.254 & 192.168 .10 .60 & \multicolumn{2}{|c|}{ GetDataValues of D101_C6100PROT/ID_PTOC1.StrVal.setMag.f } \\
\hline 192.168.10.254 & 192.168 .10 .60 & \multicolumn{2}{|c|}{ GetSGCBValues of D101_C6100PROT/LLN0.SGCB } \\
\hline 192.168.10.254 & 192.168 .10 .60 & \multicolumn{2}{|c|}{ SelectActiveSG of D101_C6100PROT/LLNO.SGCB.ActSG } \\
\hline 192.168.10.254 & 192.168 .10 .60 & \multicolumn{2}{|c|}{ GetSGCBValues of D101_C6100PROT/LLNO.SGCB } \\
\hline 192.168.10.254 & 192.168 .10 .60 & \multicolumn{2}{|c|}{ GetDataValues of D101_C6100PROT/ID_PTOC1.StrVal.setMag.f } \\
\hline 192.168.10.254 & $\mid 192.168 .10 .60$ & \multicolumn{2}{|c|}{ Release with 192.168 .10 .60} \\
\hline$\triangle$ Request & \multicolumn{2}{|c|}{ Setting Data Ref. } & $\Delta$ Response \\
\hline $\begin{array}{ll}\text { Success: } & \text { true } \\
\text { Timestamp: } 2018-0 \\
\text { Paths: } \\
\quad \text { Path: D101_C610 }\end{array}$ & \multicolumn{2}{|c|}{$\begin{array}{l}\text { true Setting Data Ref. } \\
\text { 2018-08-27 AM 4:04: }\end{array}$} & $\begin{array}{l}\text { Success: } \quad \text { true } \\
\text { Timestamp: } 2018-08-27 \text { AM 4:04:32.137 UTC } \\
\text { Values: } \\
\text { Float: } 2000 \quad \text { Setting Value }\end{array}$ \\
\hline
\end{tabular}

(d)

Figure 8. (a) SelectActiveSG (SG\#1) Test results; (b) GetDataValues (SG\#1) Test Results; (c) SelectActiveSG (SG\#2) Test Results; (d) GetDataValues (SG\#2) test results.

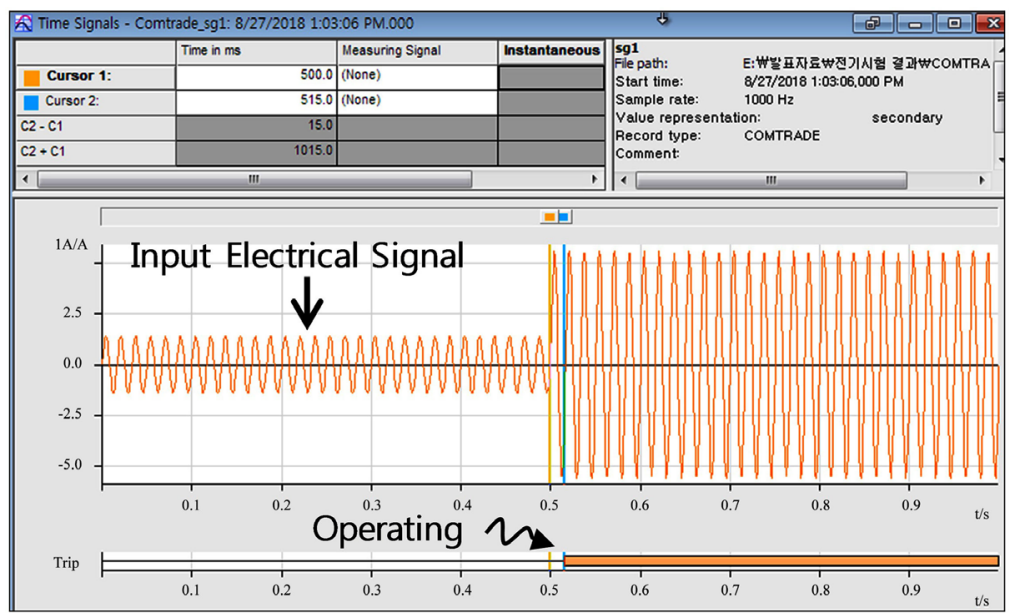

(a)

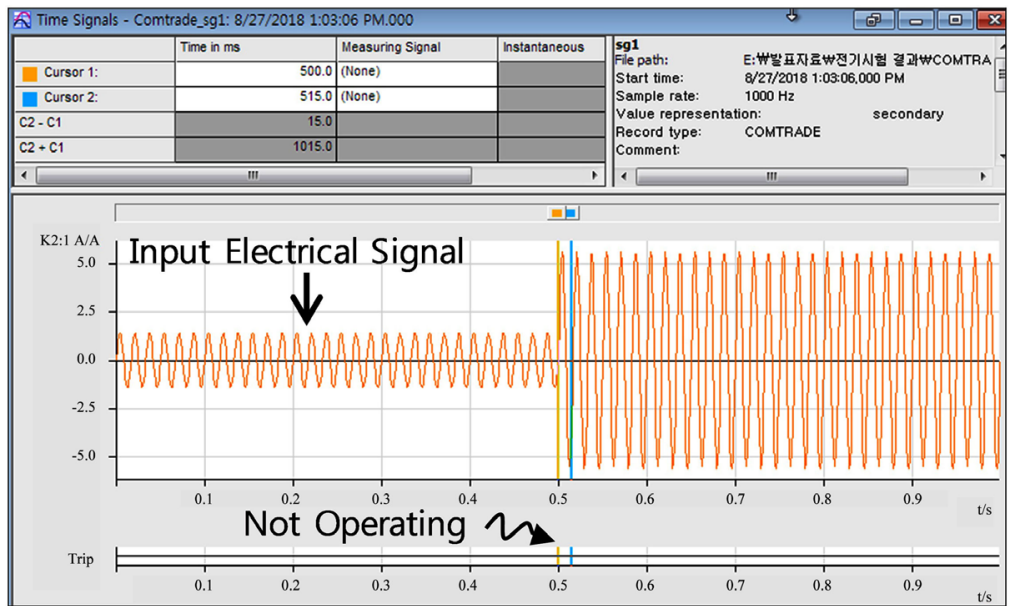

(b)

Figure 9. (a) Result of Test procedure No. 4 (SG\#1); (b) Result of Test procedure No. 8 (SG\#2). 
Table 4. Setting Group Values used for the test implementations.

\begin{tabular}{cccc}
\hline Setting Data & $\begin{array}{c}\text { Setting Group 1 } \\
\text { (SG\#1) }\end{array}$ & $\begin{array}{c}\text { Setting Group 2 } \\
\text { (SG\#2) }\end{array}$ & Operating value \\
\hline $\begin{array}{c}\text { PTOC1.StrVal.setMag.f } \\
\text { :Minimum Operating current }\end{array}$ & $800[\mathrm{~A}]$ & $2000[\mathrm{~A}]$ & $1600[\mathrm{~A}]$ \\
\hline
\end{tabular}

\section{Conclusion}

The Setting Group function, which is expected to be introduced into the intelligent digital substation system, does not require the IED to be completely re-engineered using manufacturer-specific engineer tool whenever a single value needs to be changed. It is possible to just change the values using the Setting Group functionality by activating a specific Setting Group and then accessing the various attributes using the standardized communication services. Before adopting or using any IEC 61850 communication service, there is the need to test the conformance of the IED in accordance to the UCAIUG conformance test procedure. Generally, this test procedure focuses on only the communication between the IED device and the conformance testing tool or a client without considering the functional test of each communication service using electrical signals. In this paper, we propose a method that can integrate the communication function and the electric functional test for the Setting Group functionality of an IED. By standardizing the integrated test procedure, the results can be made global and easily reproducible. The communication test can be verified using the protocol analyzers and the electrical functional tests can be verified using any electrical fault recording and analysis tool like SIGRA 4 . This can assist test engineers to not only verify the request-response of the IED as requested by the host operating service but to also confirm is the IED operated normally in a practical substation where an external electrical signal is applied. This work is important because it simplifies the procedure for testing both electrical impulses and communication signals in a typical digital substation. The operator, having an understanding of the IEC 61850 communication service testing does not need to completely turn down an IED in order to implement an electrical testing but can utilize the conformance testing function to check the electrical responsiveness of the IED. In the future, we plan to study how to automate the integrated functional test procedure by adding an application program interface (API) module that can operate the current and voltage application simulator to the server conformance test tool developed by KEPCO.

\section{Conflicts of Interest}

The authors declare no conflicts of interest regarding the publication of this paper.

\section{References}

[1] Brunner, C. (2008) The Impact of IEC 61850 on Protection. Proceedings of the IET 9th International Conference on Developments in Power System Protection (DPSP 
2008), Glasgow, 17-20 March 2008, 14-19. https://doi.org/10.1049/cp:20080003

[2] Horalek, J., Matyska, J. and Sobeslav, V. (2013) Communication Protocols in Substation Automation and IEC 61850 Based Proposal. Proceedings of the IEEE 14th International Symposium on Computational Intelligence and Informatics (CINTI), Budapest, 19-21 November 2013, 321-326.

https://doi.org/10.1109/CINTI.2013.6705214

[3] Huang, W. (2018) Learn IEC 61850 Configuration in 30 Minutes. Proceedings of the 71 st Annual Conference for Protective Relay Engineers (CPRE), College Station, TX, 26-29 March 2018, 1-5. https://doi.org/10.1109/CPRE.2018.8349803

[4] Gupta, R.P. (2008) Substation Automation Using IEC61850 Standard. Proceedings of the 15th National Power Systems Conference (NPSC), IIT Bombay, Mumbai, 16-18 December 2008.

http://www.krec.ir/Automation/Substation_Automation_Using_IEC61850_Standar d.pdf

[5] Roostaee, S., Hooshmand, R. and Ataei, M. (2011) Substation Automation System Using IEC 61850. Proceedings of the 5 th International Power Engineering and Optimization Conference, Shah Alam, Selangor, 6-7 June 2011, 393-397. https://doi.org/10.1109/PEOCO.2011.5970443

[6] Khavnekar, A., Wagh, S. and More, A. (2015) Comparative Analysis of IEC 61850 Edition-I and II Standards for Substation Automation. Proceedings of the IEEE International Conference on Computational Intelligence and Computing Research (ICCIC), Madurai, 10-12 December 2015, 1-6. https://doi.org/10.1109/ICCIC.2015.7435756

[7] Apostolov, A. (2017) Impact of IEC 61850 Edition 2 on the Object Modelling of Distribution Protection IEDs. CIRED-Open Access Proceedings Journal, 2017, 1184-1187. https://doi.org/10.1049/oap-cired.2017.0495

[8] Schossig, T. (2018) Application of Settings and Setting Groups in IEC 61850. The Journal of Engineering, 2018, 967-970. https://doi.org/10.1049/joe.2018.0191

[9] Lee, N.H., Jang, B.T. and Song, I.J. (2009) The Testing Method of Data Transmission Services of IEC 61850 Based Client. Proceedings of the Transmission \& Distribution Conference \& Exposition: Asia and Pacific, Seoul, 26-30 November 2009, 1-4. https://doi.org/10.1109/TD-ASIA.2009.5356912

[10] Choi, C., Park, J., Han, J., Shin, Y., Kim, Y. and Shim, E. (2011) The Development of a Client Conformance Tool Based on IEC 61850. Proceedings of the International Conference on Electrical Machines and Systems, Beijing, 20-23 August 2011, 1-5. https://doi.org/10.1109/ICEMS.2011.6073718

[11] Muschlitz, B. (2006) IEC 61850 Conformance Testing: Goals, Issues and Status EnerNex Corporation. Presented at DistribuTECH 2006, Tampa, Florida Feb. 7-9 2016. http://romvchvlcomm.pbworks.com/f/Muschlitz61850conftest.pdf

[12] Udren, E.A., Strabbing, W. and Dolezilek, D. (2007) "IEC 61850: Role of Conformance testing in Successful Integration. Proceedings of the 19th International Conference and Exhibition on Electricity Distribution, Vienna, 21-24 May 2007.

[13] (2013) Communication Networks and Systems for Power Utility Automation Part 7-1: Basic Communication Structure Principles and Models, IEC 61850 Standard, 2013.

[14] (2013) Communication Networks and Systems for Power Utility Automation Part 7-2: Basic Communication Structure-Abstract Communication Service Interface (ACSI), IEC 61850 Standard, 2013.

[15] Schimmel, R. (2013) Conformance Test Procedures for Server Devices with IEC 61850-8-1 Edition 2 Interface-Revision 1. 